\title{
Systemic combinatorial peptide selection yields a non-canonical iron-mimicry mechanism for targeting tumors in a mouse model of human glioblastoma
}

Fernanda I. Staquicini, ${ }^{1}$ Michael G. Ozawa, ${ }^{1}$ Catherine A. Moya, ${ }^{1}$ Wouter H.P. Driessen, ${ }^{1}$ E. Magda Barbu, ${ }^{1}$ Hiroyuki Nishimori, ${ }^{2}$ Suren Soghomonyan, ${ }^{3}$ Leo G. Flores 2 nd, ${ }^{3}$ Xiaowen Liang, ${ }^{4}$ Vincenzo Paolillo, ${ }^{3}$ Mian M. Alauddin, ${ }^{3}$ James P. Basilion, ${ }^{5}$ Frank B. Furnari, ${ }^{2}$ Oliver Bogler, ${ }^{6}$ Frederick F. Lang, ${ }^{6}$ Kenneth D. Aldape, ${ }^{7}$ Gregory N. Fuller, ${ }^{7}$ Magnus Höök, ${ }^{4}$ Juri G. Gelovani, ${ }^{3}$ Richard L. Sidman, ${ }^{8}$ Webster K. Cavenee, ${ }^{2}$ Renata Pasqualini, ${ }^{1,3}$ and Wadih Arap ${ }^{1,3}$

1David H. Koch Center, The University of Texas M.D. Anderson Cancer Center, Houston, Texas, USA. ${ }^{2}$ Ludwig Institute for Cancer Research, University of California San Diego, La Jolla, California, USA. ${ }^{3}$ Department of Experimental Diagnostic Imaging, The University of Texas M.D. Anderson Cancer Center, Houston, Texas, USA. ${ }^{4}$ Texas A\&M University System Health Science Center, Houston, Texas, USA. ${ }^{5}$ Center for Molecular Imaging Research and National Foundation for Cancer Research (NFCR)

Center for Molecular Analysis and Imaging, Department of Radiology, Massachusetts General Hospital, Boston, Massachusetts, USA. ${ }^{6}$ Department of Neurosurgery and ${ }^{7}$ Department of Pathology, The University of Texas M.D. Anderson Cancer Center, Houston, Texas, USA. ${ }^{8}$ Department of Neurology, Beth Israel Deaconess Medical Center, Harvard Medical School, Boston, Massachusetts, USA.

\begin{abstract}
The management of CNS tumors is limited by the blood-brain barrier (BBB), a vascular interface that restricts the passage of most molecules from the blood into the brain. Here we show that phage particles targeted with certain ligand motifs selected in vivo from a combinatorial peptide library can cross the BBB under normal and pathological conditions. Specifically, we demonstrated that phage clones displaying an ironmimic peptide were able to target a protein complex of transferrin and transferrin receptor (TfR) through a non-canonical allosteric binding mechanism and that this functional protein complex mediated transport of the corresponding viral particles into the normal mouse brain. We also showed that, in an orthotopic mouse model of human glioblastoma, a combination of TfR overexpression plus extended vascular permeability and ligand retention resulted in remarkable brain tumor targeting of chimeric adeno-associated virus/ phage particles displaying the iron-mimic peptide and carrying a gene of interest. As a proof of concept, we delivered the HSV thymidine kinase gene for molecular-genetic imaging and targeted therapy of intracranial xenografted tumors. Finally, we established that these experimental findings might be clinically relevant by determining through human tissue microarrays that many primary astrocytic tumors strongly express TfR. Together, our combinatorial selection system and results may provide a translational avenue for the targeted detection and treatment of brain tumors.
\end{abstract}

\begin{abstract}
Introduction
Historically, the blood-brain barrier (BBB) has been considered the major impediment to the systemic treatment of CNS diseases $(1,2)$. Access to the brain via its capillaries is obstructed by the neurovascular unit formed by endothelial cells, associated astrocytic "endfeet" processes, and their shared basement membrane, perivascular neurons, and pericytes (3-6). Although all 4 cell types contribute to the functioning of the brain microvasculature, only the capillary endothelial cells are thought to control permeability (7). In contrast to other organs, tight junctions formed between CNS endothelial cells render the brain and spinal cord practically inaccessible to circulating polar molecules, unless specific transport pathways at the BBB transfer them.
\end{abstract}

\footnotetext{
Authorship note: Fernanda I. Staquicini, Michael G. Ozawa, and Catherine A. Moya contributed equally to this work.

Conflict of interest: The University of Texas and some of its researchers (Wadih Arap and Renata Pasqualini) have equity in Mercator Therapeutics, which is subjected to certain restrictions under university policy; the university manages and monitors the terms of these arrangements in accordance to its conflict-of-interest policies. Citation for this article: J Clin Invest. 2011;121(1):161-173. doi:10.1172/JCI44798.
}

To gain insight into targeting the CNS, we used the combinatorial approach afforded by selection of phage display peptide libraries in vivo. A few general mechanisms are known by which molecules in the bloodstream can gain access to brain interstitial fluid: (a) the extracellular pathways (8), (b) peptide transporter systems (9), (c) lipid-mediated transport of lipid-soluble small molecules with molecular weights of less than $400 \mathrm{Da}(3,4)$, and (d) facilitated transport, including carrier-mediated transport for small molecules, receptor-mediated transport (RMT) for large molecules $(3-5,10,11)$, and absorptive endocytosis $(12,13)$.

Among the best established of such membrane transporters on CNS capillary endothelial cells are receptors for transferrin (Tf), insulin, leptin, and low-density lipoprotein (3-7, 10, 11, 14-19). Given that the Tf receptor (TfR) has the highest gene expression ratio in human glioblastoma compared with normal brain (Supplemental Table 1; supplemental material available online with this article; doi:10.1172/ JCI44798DS1), we reasoned that an unbiased targeting of CNS would likely yield ligands to this particular RMT. Indeed, analysis of our selection in vivo uncovered candidate motifs targeting the Tf/TfR system, along with what we believe to be a new molecular mechanism. 
Here we introduce, optimize, and validate this translational approach for CNS delivery of agents and genes based on an endogenous BBB endothelial cell receptor, the engagement of which is followed by endocytosis and release into the parenchyma of the normal brain or brain tumors. We demonstrate that a targeted phage displaying the cyclic peptide CRTIGPSVC crosses the intact $\mathrm{BBB}$. We identify the underlying molecular pathways under both normal and pathological conditions. In vitro, we show that the targeting peptide CRTIGPSVC selectively interacts with apotransferrin (apo-Tf) to induce allosteric conformational changes that functionally "mimic" iron through a non-canonical liganddirected mechanism. In vivo, we present evidence for specific associations that lead to binding, internalization, and transcytosis of targeted phage particles into the normal mouse brain and even more strongly into xenografts of human malignant glioma. Such intrinsic properties were further explored for the development of an integrated, targeted molecular-genetic imaging and treatment (so called, "see and treat") of intracranial tumors. We used a vector with genomic cis-elements of adeno-associated virus (AAV) and of M13-derived phage (termed AAV phage [AAVP]) (20-23) to display the CRTIGPSVC peptide. We show that the CRTIGPSVC-targeted AAVP-HSV-TK, when systemically delivered into brain tumor-bearing mice (a) allows molecular imaging of a reporter transgene, (b) enables serial noninvasive monitoring of intracranial tumors in a preclinical setting, and (c) allows ligand-directed targeting and vascular ablation of human malignant glioma xenografts through a suicide gene approach. Finally, we confirm the clinical potential of our results in a comprehensive panel of human malignant glioma tissue microarray (TMA) samples. Taken together, we believe these findings present a novel, readily accessible, and effective candidate strategy for detection, treatment, and monitoring of human brain tumors and possibly other CNS disorders.

\section{Results}

Receptor-mediated delivery of targeted phage particles across intact $B B B$. We used a phage-displayed library of random $\mathrm{CX}_{7} \mathrm{C}$ peptides (C, cysteine; X, any residues) (24-26) to enrich serially for motifs that bind selectively to CNS endothelial cells and are then internalized and transported across the intact BBB in vivo. We administered the phage library i.v. into normal mice (26) and recovered the enriched population of brain-homing phage after 3 rounds of selection. We used bioinformatics to identify candidate motifs and to minimize or eliminate background for further evaluation $(24,25)$ and applied a basic local alignment and search tool (BLAST) of selected sequences online (National Center for Biotechnology Information/PubMed; http://blast.ncbi.nlm.nih.gov/) to detail protein alignment and to identify peptides with high similarity to Tf. More than 30 nonredundant peptide sequences ( $20 \%$ of the total) showed similarity to Tf and were further analyzed. We identified 3 main clusters of candidate Tf-like motifs enclosed within the $\mathrm{N}$-lobe of Tf: clusters 1 and 3 matched to 2 distinct segments of domain $\mathrm{N} 1$ (at the $\mathrm{N}$ and $\mathrm{C}$ termini), while cluster 2 was centered within domain N2 (Figure 1A). 3D models of the iron-bound Tf N-lobe (ref. 27 and Figure 1B; residues 1-331) and iron-bound Tf complexed to TfR (ref. 28 and Figure 1C) positioned at cluster 2 around the iron atom, within the Tf iron-binding cleft. Clusters 1 and 3 were located on the exposed surface of Tf. We focused our functional analysis on the cyclic targeting peptide CRTIGPSVC, originally grouped in cluster 1 . The remaining candidate peptides were not functionally pursued here.
To evaluate the targeting attributes of the selected peptide in vivo, we administered the CRTIGPSVC-phage i.v. into normal mice and first assessed homing by recovery from tissue homogenates, followed by counting of transducing units (TU) (data not shown) and quantitative real-time PCR (Figure 1D). Non-targeted (insertless) phage and homing to control organs (muscle is shown) served as negative controls (Figure $1 \mathrm{D}$ and Supplemental Figure 1, A-C). We administered phage i.v. into tail veins of mice; surgically collected brains and control organs 10 minutes, 30 minutes, and 24 hours later; and amplified phage DNA. We detected approximately 100-fold more CRTIGPSVC-phage particles in the normal brain after a 10-minute circulation compared with those of insertless phage. A similar result was obtained at 30 minutes, with a reduction to approximately 40 -fold difference detected at 24 hours after administration (Figure 1D). Homing of targeted and insertless phage to control organs (Supplemental Figure 1, A-C), including bone marrow (Supplemental Figure 2), was at background levels. Preclinical pharmacokinetic studies showed no significant differences in the half-lives of targeted and control phage in the blood of normal mice (Supplemental Figure 3A), suggesting that superior uptake of targeted phage in the brain is mediated by specific ligand-receptor interactions. Lastly, separation of brain microvessels from the brain parenchyma $(14,29,30)$ confirmed the ability of the CRTIGPSVC-phage to cross the intact BBB (Figure 1E). Normal mice were i.v. injected with targeted or control phage. Brains were collected after 10 minutes, 30 minutes, and 24 hours and homogenized and fractionated on a gradient of $30 \%$ dextran solution (w/v). Quantitative real-time PCR demonstrated the presence of CRTIGPSVC-phage in both fractions (blood vessels and blood vessel-depleted brain parenchyma). Insertless phage was not detected in the parenchyma fraction (Figure 1E). Real-time PCR confirmed partial purity of the isolated fractions using capillaryand endothelial cell-specific genes (Supplemental Figure 3B).

The targeting peptide CRTIGPSVC binds to Tf/TfR through an allosteric mechanism. Having shown that targeted phage particles displaying the Tf-mimic CRTIGPSVC peptide crossed the intact BBB, we next attempted to elucidate the molecular mechanism(s) mediating its transport from blood into brain. First, to establish whether CRTIGPSVC is indeed a mimic of Tf, we evaluated the binding of CRTIGPSVC-phage to cells overexpressing human TfR (ref. 31 and Figure 1F). To determine binding specificity, we tested the effects of apo-Tf (iron-free, open conformation) and holo-Tf (iron-containing, closed conformation) on the binding of phage to the cell surface. Unexpectedly, we observed enhanced binding when apo-Tf was added to the system (Student's $t$ test, $P<0.05$ ). Next, we analyzed the binding of CRTIGPSVC-phage to TfR and to apo-Tf and holo$T f$ in a cell-free system. Insertless phage and BSA were used as negative controls. Consistently, we observed binding of CRTIGPSVCphage to apo-Tf but not to TfR or holo-Tf (Figure 1G).

Given that CRTIGPSVC-phage binds selectively to apo-Tf, we hypothesized that the CRTIGPSVC peptide might functionally mimic iron. If so, one can speculate that binding of CRTIGPSVC, like binding of iron, would induce allosteric conformational changes in apo-Tf, leading to endocytosis and ultimately to release of peptide-targeted phage into the cytoplasm of TfR-positive cells. To test this hypothesis, we designed a series of phage binding assays in vitro to apo-Tf and holo-Tf in the presence of ferric iron $\left(\mathrm{Fe}^{+3}\right)$ and EDTA (Figure 1, H and I). Iron abrogated binding of CRTIGPSVC-phage to apo-Tf (Figure 1H), whereas no major alterations were observed in the binding of targeted phage 


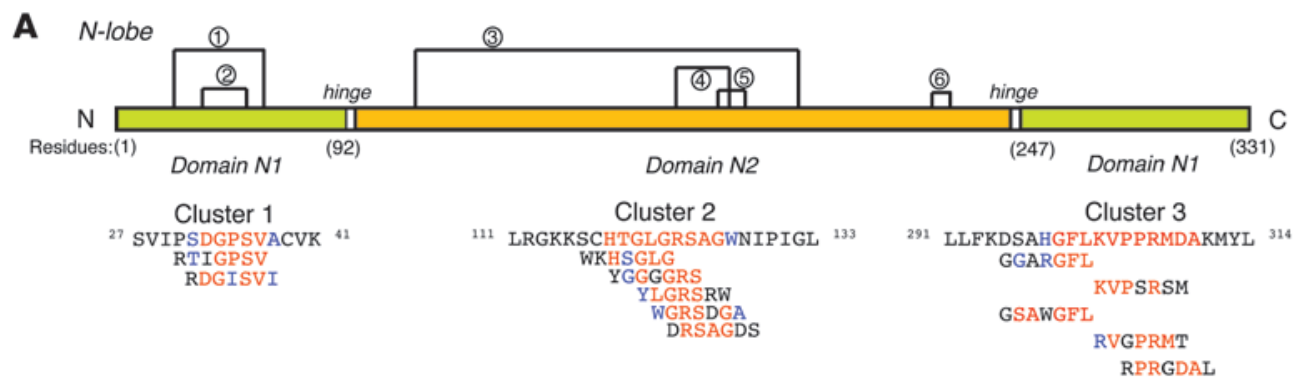

B

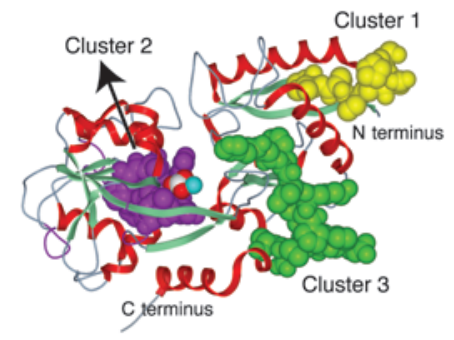

D

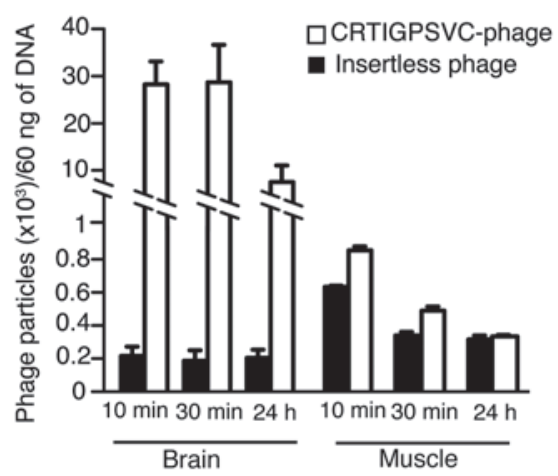

G

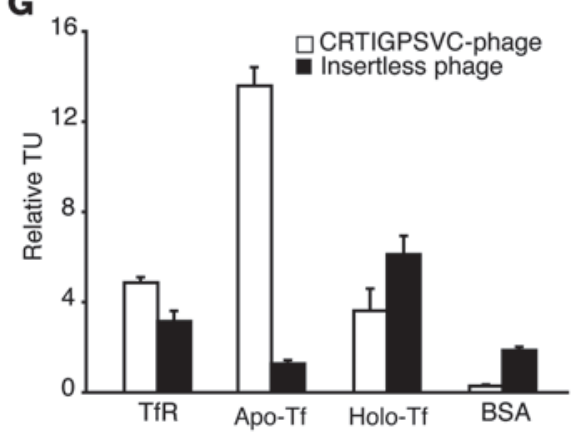

C $N$-lobe Cluster $2 \quad \mathrm{~N}$-lobe

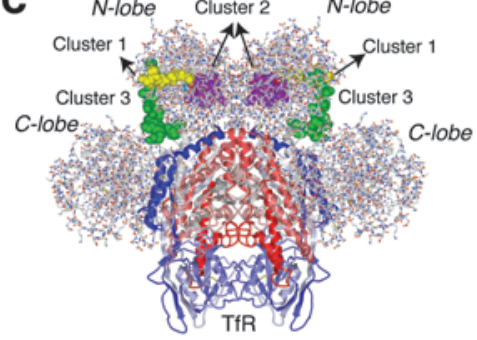

E

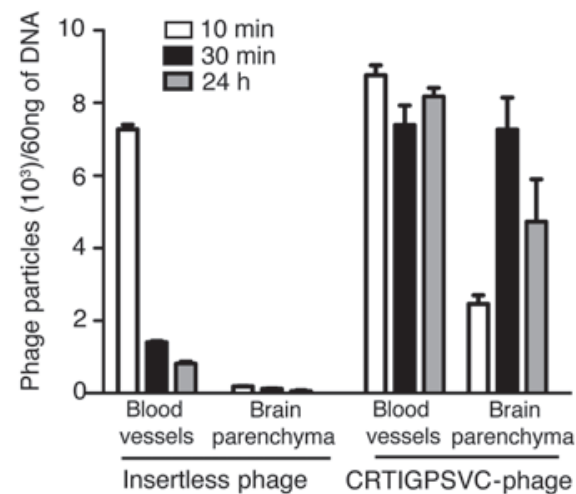

H

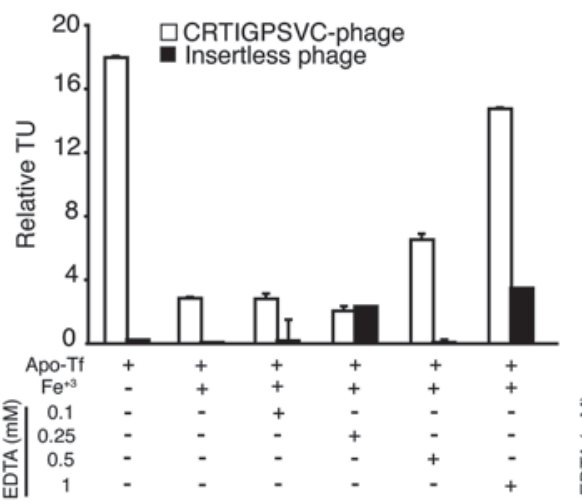

F

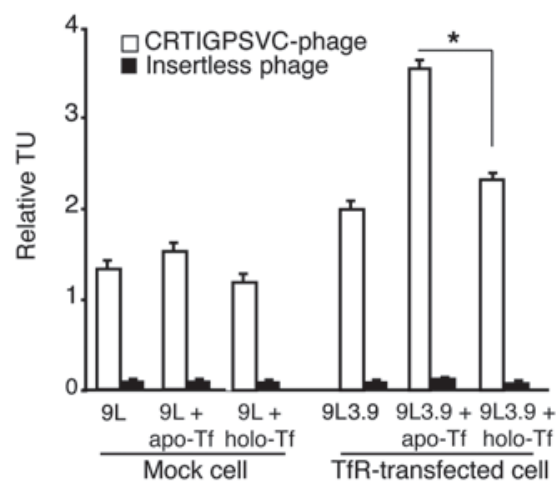

I

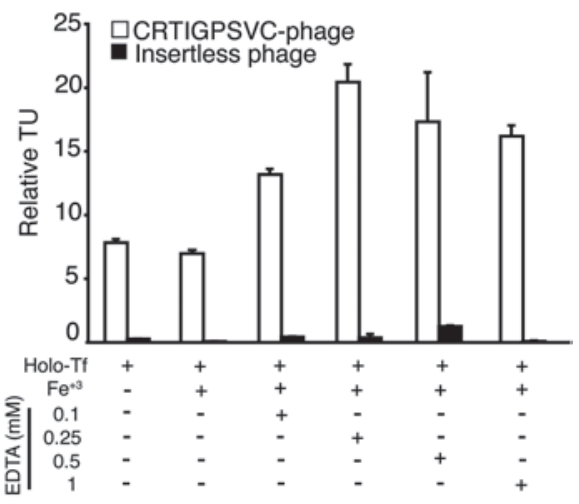

Figure 1

Targeting the specificity of phage displaying the peptide CRTIGPSVC in normal mouse brain. (A) Overall structure of the N-lobe half-molecule of Tf. Domains N1 and N2 are indicated. The numbers represent the 6 consensus disulfide bonds, Cys10-Cys49, Cys20-Cys40, Cys 119-Cys195, Cys159-Cys175, Cys162-Cys180, and Cys172-Cys178, respectively. (B) Ribbon diagram showing the structural organization of iron-loaded $\mathrm{Tf}$ and location of the peptide clusters. (C) Side view of the Tf/TfR molecular complex. $\mathrm{N}$ - and C-lobes are shown. (D) Homing specificity of CRTIGPSVC-phage and insertless phage in normal brain compared with that in non-brain control organ (muscle is shown). CRTIGPSVC-phage and insertless phage were administered i.v. into mice. Phage was allowed to circulate for 10 minutes, 30 minutes, and 24 hours, and tissues were recovered and processed as described in Methods. (E) Fractionation of brain microvessels and parenchyma confirmed the ability of the CRTIGPSVC-phage to cross the intact BBB. (F) Binding of phage to rat gliosarcoma 9L cells and 9L cells expressing human TfR (9L3.9) in the presence of apo-Tf and holo-Tf. ${ }^{*} P<0.05$. (G) Binding assays reveal that CRTIGPSVC-phage target human apo-Tf in vitro. Insertless phage and BSA served as negative controls. (H and I) The effect of iron on the binding of phage to apo-Tf was reversed by EDTA, but no effect was observed with holo-Tf. In vitro binding assays were performed in 96-well plates coated with either human apo-Tf $(\mathbf{H})$ or with human holo-Tf (I), in the presence of iron and increasing amounts of EDTA. 
A

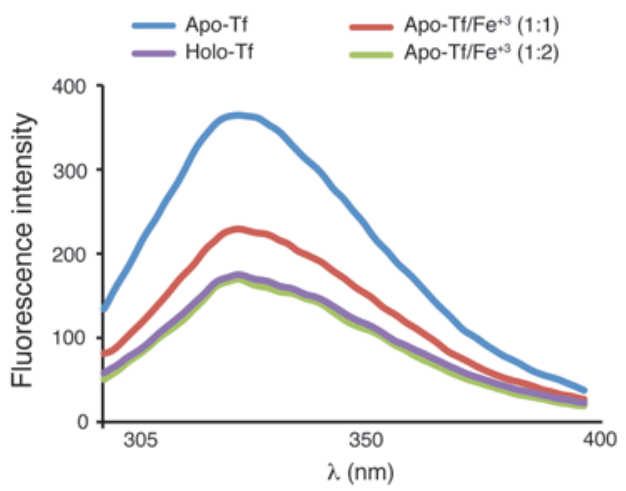

C

$\begin{array}{ll}\text { Apo-Tf } \\ \text { Holo-Tf } \\ \text { Apo-Tf/control peptide 1 (1:6) } & \text { Apo-Tf/control peptide } 2(1: 6) \\ \text { Apo-Tf/control peptide } 3(1: 6) \\ \text { Apo-Tf/control peptide } 4(1: 6)\end{array}$

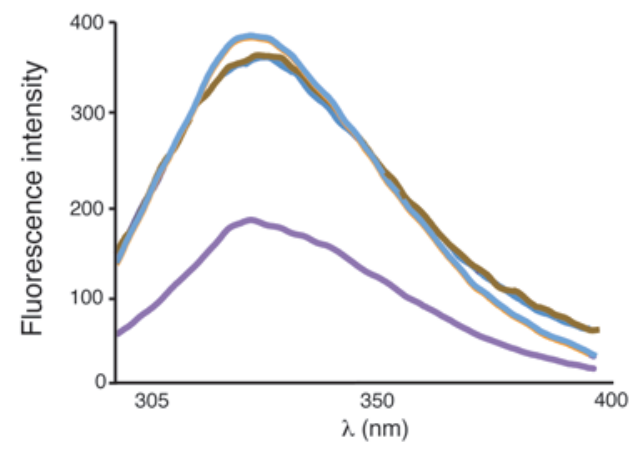

E

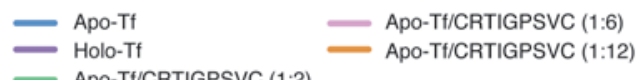

- Apo-Tf/CRTIGPSVC (1.2)
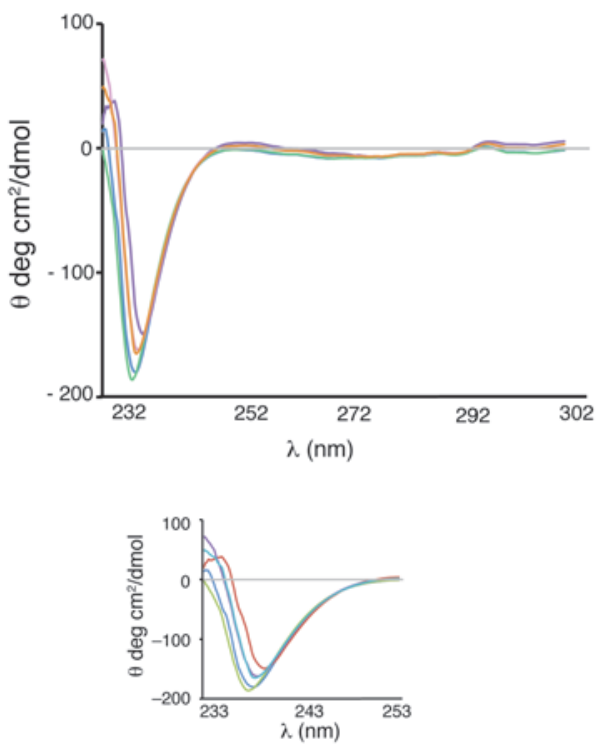

B

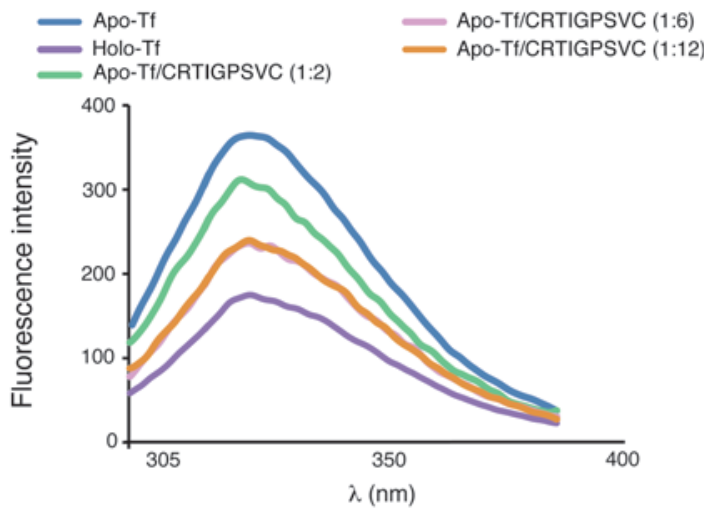

D
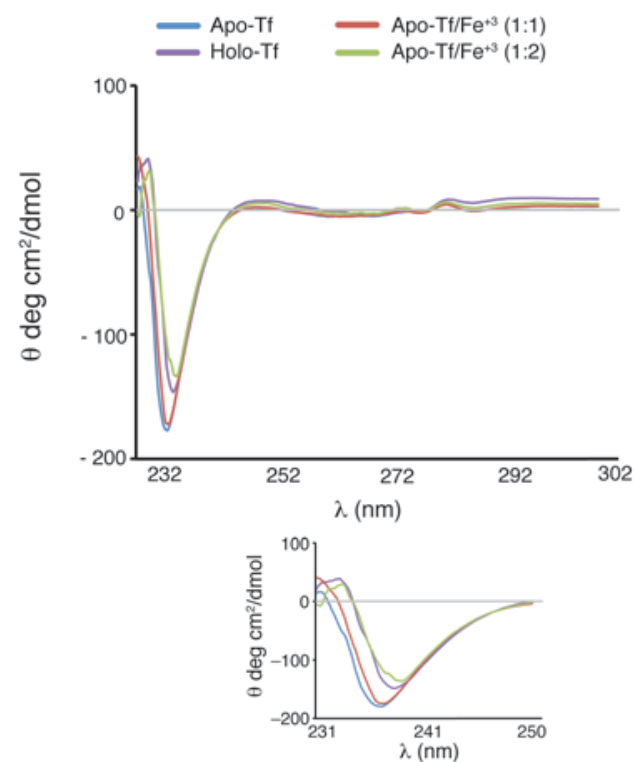

$\mathbf{F}$
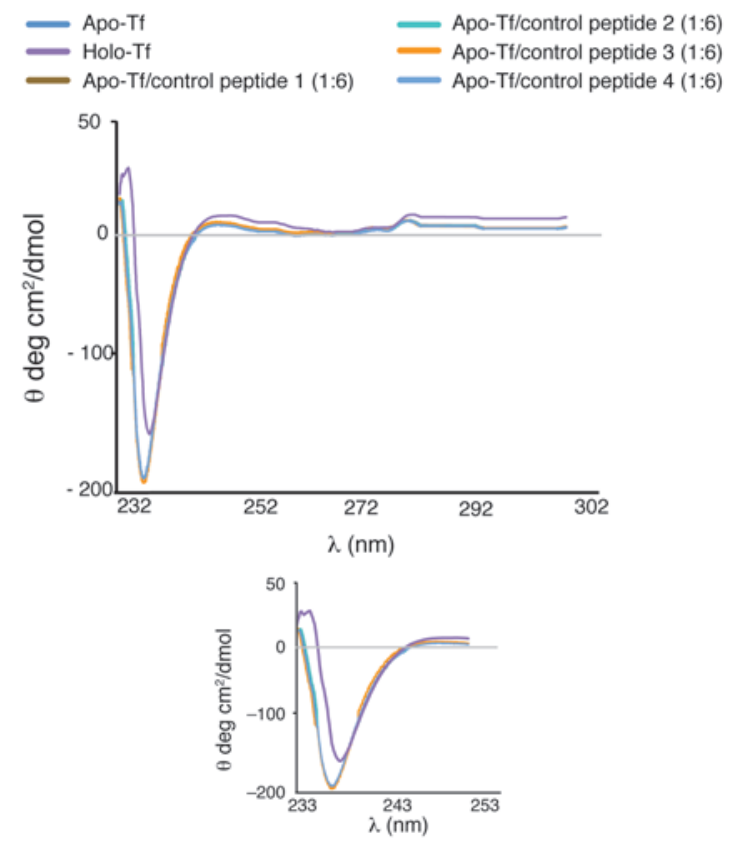


\section{Figure 2}

CRTIGPSVC peptide binds to human apo-Tf and induces allosteric conformational changes. (A-C) Steady-state emission spectra of (A) apoTf in presence of iron or (B) distinct concentrations of the CRTIGPSVC peptide. (C) Four different peptide sequences were used as control (control peptide 1, CGLPYSSVC; control peptide 2, CSGIGSGGC; control peptide 3, CRFESSGGC; and control peptide 4, CPQRGVTPC). (D-F) Near-UV CD spectra of (D) apo-Tf in presence of iron or (E) distinct concentrations of the CRTIGPSVC peptide. (F) Four different peptide sequences were used as control (control peptide 1, CGLPYSSVC; control peptide 2, CSGIGSGGC; control peptide 3, CRFESSGGC; and control peptide 4, CPQRGVTPC). Insets show the $\mathrm{CD}$ spectral region between 230 and $250 \mathrm{~nm}$.

to holo-Tf (Figure 1I). Consistently, the effect of iron on the binding of peptide-targeted phage particles to apo-Tf or holo-Tf was reversed by the addition of EDTA (Figure 1, H and I), an indication that CRTIGPSVC and iron have similar allosteric effects on apoTf. The same results were obtained with the CRTIGPSVC synthetic peptide, increasing concentrations of which abrogated binding of targeted phage to apo-Tf (data not shown). These results suggest that CRTIGPSVC-phage functionally mimics iron and binds to apo-Tf. Moreover, these data are consistent with the hypothesis that selective internalization of the Tf/TfR/CRTIGPSVC complex into TfR-positive cells is mediated by conformational changes induced by CRTIGPSVC binding to apo-Tf.

To evaluate this working hypothesis, we applied optical methodologies, initially using analysis by steady-state fluorescence (refs. 32-34 and Figure 2, A-C). First, we assigned changes in fluorescence spectra to apo-Tf in the absence or presence of $\mathrm{Fe}^{3+}$ (Figure 2A). Next, we used increasing concentrations of the CRTIGPSVC peptide to evaluate changes in apo-Tf conformation as indicated by changes in fluorescence spectra. We observed a gradual shift of the apo-Tf fluorescence spectra in the presence of increasing amounts of CRTIGPSVC (Figure 2B); under these experimental conditions, the interaction reached saturation at 1:6 molar ratio (apo-Tf/CRTIGPSVC). Finally, a panel of unrelated control peptides served to demonstrate that the phenomenon is specific to the interaction between apo-Tf and CRTIGPSVC (Figure 2C). Consistent experimental results were also obtained with another optical method, circular dichroism (CD) analysis (Figure 2, D-F). As expected, adding $\mathrm{Fe}^{+3}$ to apo-Tf generated a shift in the spectra, reflecting the known iron-induced conformation change from apo-Tf to holo-Tf (refs. 35-37 and Figure 2D). The CRTIGPSVC peptide also mediated conformation changes in apo-Tf (Figure 2E), with no such changes detected with the addition of unrelated control peptides (Figure 2F). Together, these data strongly suggest that the CRTIGPSVC peptide mediates a protein conformational change in apo-Tf. One should note that, unlike iron, the peptide does not appear to mediate a full conformational change from apo-Tf to holo-Tf. Thus, further studies will be required to understand the precise nature of this peptide-protein interaction.

CRTIGPSVC-phage targets Tf/TfR in vivo. Because CRTIGPSVCphage binds to apo-Tf in vitro and the CRTIGPSVC peptide changes the conformation of apo-Tf in a concentration-dependent manner, we next asked whether the same mechanism would be implicated in the homing and transport of phage particles across the BBB in vivo. Because the overexpression of TfR has been demonstrated in primary brain tumors (refs. 38-40 and Supplemental
Table 1), we chose to study the targeting properties of CRTIGPSVCphage in an orthotopic model of human-derived glioblastoma multiforme xenografts (ref. 41 and Figures 3 and 4).

First, we compared the expression of TfR in normal brains and in a model of human glioma xenografts (Supplemental Figure 4). CD31 (PECAM-1) was used as a standard vascular marker (Supplemental Figure 4, A and D). While the expression of TfR was moderate and restricted to blood vessels in the normal brain (Supplemental Figure 4, A-C), we observed expression of TfR in both tumor blood vessels (arrows) and tumor stroma (arrowheads) (Supplemental Figure 4, $\mathrm{D}$ and $\mathrm{F}$ ). The result establishes human-derived malignant glioma xenografts as an appropriate experimental system for the study of $\mathrm{Tf} / \mathrm{TfR} /$ peptide-mediated phage targeting and delivery in vivo.

The binding capacity and targeting properties of the CRTIGPSVCphage were subsequently compared in normal tissue and tumor (Figures 3 and 4). Tumor-bearing animals received phage i.v., and tumor and contralateral brain hemisphere (normal tissue) were collected after 10 minutes, 30 minutes, and 1 hour (Figure 3). Phage DNA was isolated and amplified from a series of coronal brain sections (each $\sim 1$-mm thick) (Figure $3 \mathrm{~A}$ ), and quantitative real-time PCR was used to measure the levels of phage particles in normal brain compared with those in tumor samples and control organs (Supplemental Figure 5, A-C).

After 10 minutes of circulation, variable levels of the CRTIGPSVCphage were observed in normal brain slices, whereas control insertless phage was not detected. In contrast, comparable levels of both targeted and insertless phage were obtained in tumor (Figure 3B); however, after 30 minutes, the same amount of targeted phage was detected in tumor, while the number of phage particles in normal brain slices was markedly reduced. Notably, insertless phage was not observed in either tumor or normal organ (Figure 3C). At 1 hour after targeted phage administration, we detected approximately $1.6 \times 10^{4} \mathrm{TU} / 60 \mathrm{ng}$ DNA in tumor (Figure 3D), whereas normal brain tissue and insertless phage showed phage DNA only at background levels. We observed phage accumulation in the spleen and liver of mice at all time points (Supplemental Figure 5, $\mathrm{A}-\mathrm{C}$ ), a result of the long-established pattern of nonspecific particle clearance in the reticuloendothelial system $(26,42,43)$. Similar experiments performed in mice bearing subcutaneous human xenografts confirmed specific homing of CRTIGPSVC-phage after systemic administration, compared with that of control phage (Supplemental Figure 5D). The number of targeted phage particles recovered in subcutaneous tumors was approximately 10 -fold lower than that obtained in intracranial tumors; this result suggests that potentially more than 1 targeting mechanism may be involved in the setting of a compromised BBB.

Clearly, a distinct phage distribution profile is obtained in brains with tumor, a result indicative that phage-targeting properties are largely dictated by receptor accessibility to the circulating ligand peptide (44). Additional established factors, such as increased vascular permeability leading to increased retention within tumors, a phenomenon known as enhanced permeability and retention effect (45-47), and increased activity of tumor endothelium (44), are likely to contribute to the observed enhanced targeting by CRTIGPSVC-directed phage particles. Future studies will be required to elucidate the relative contribution of each individual non-mutually exclusive mechanism(s) in the peptidemediated brain tumor selectivity.

Immunohistochemistry revealed specific homing of phage to the tumor vasculature (Figure 4A). A strong immunostaining 
A

(1)

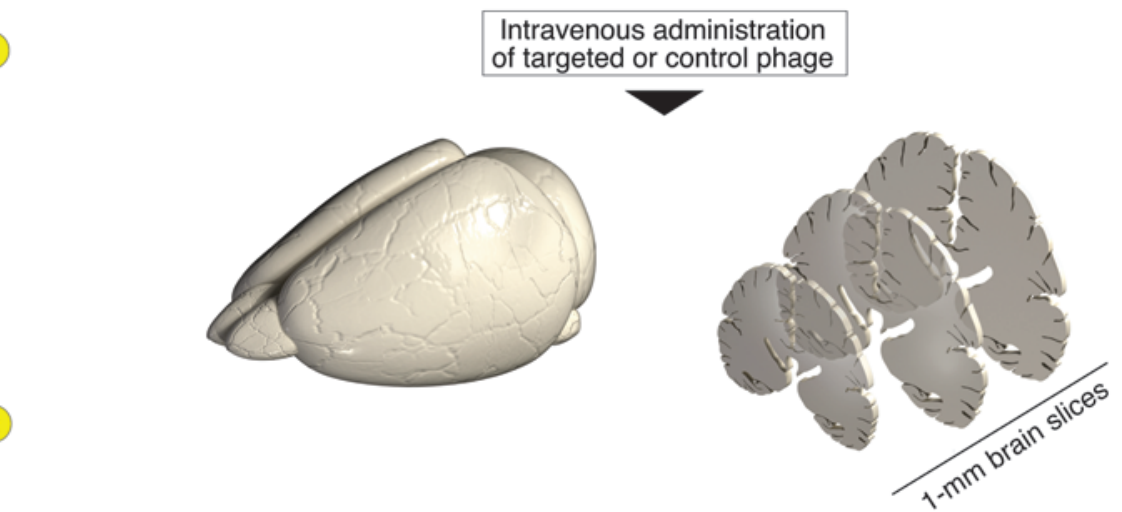

(2)

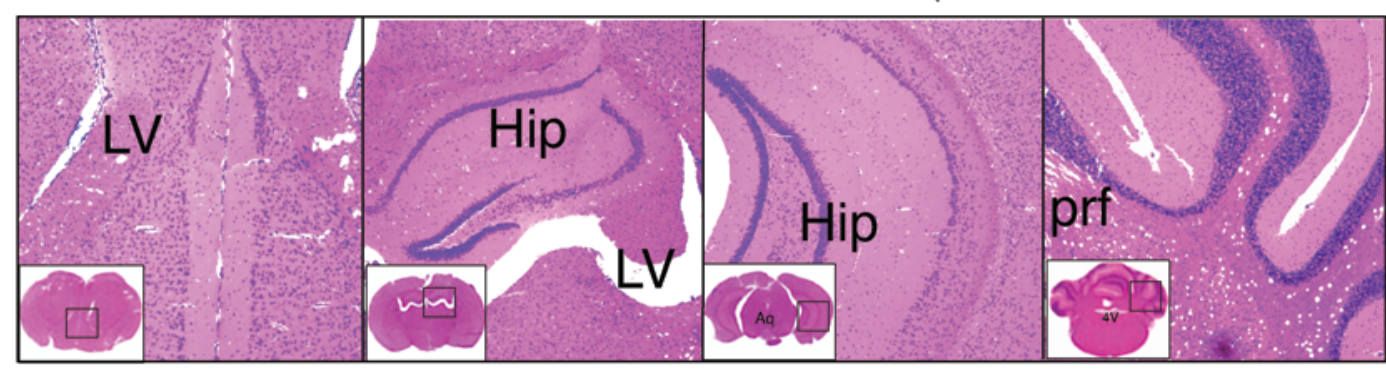

(3)

Slice 2

Slice 4

Slice 6

Slice 9

Quantitative real-time PCR
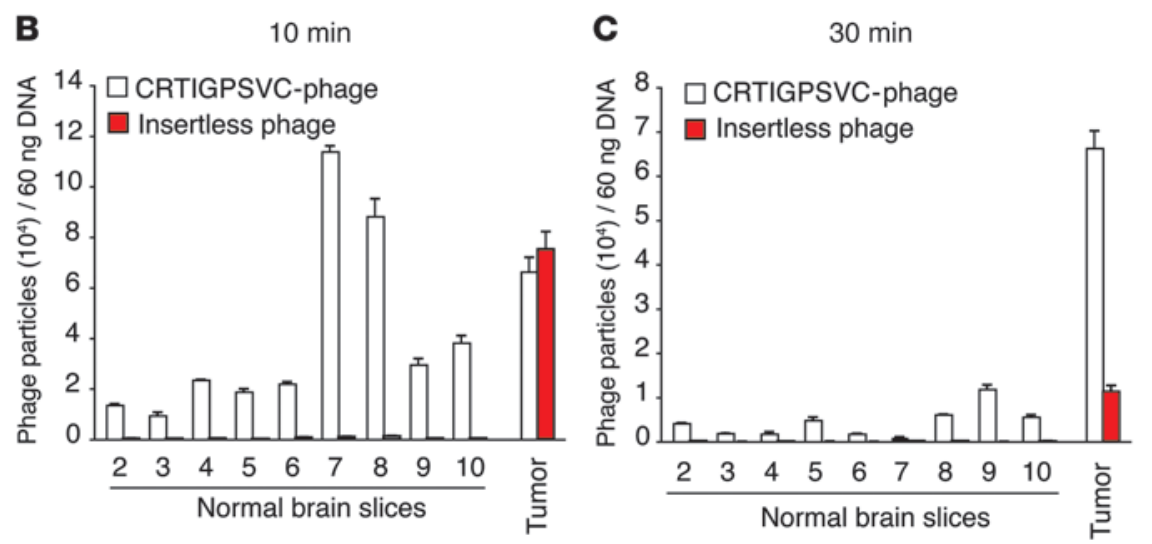

D

$1 \mathrm{~h}$

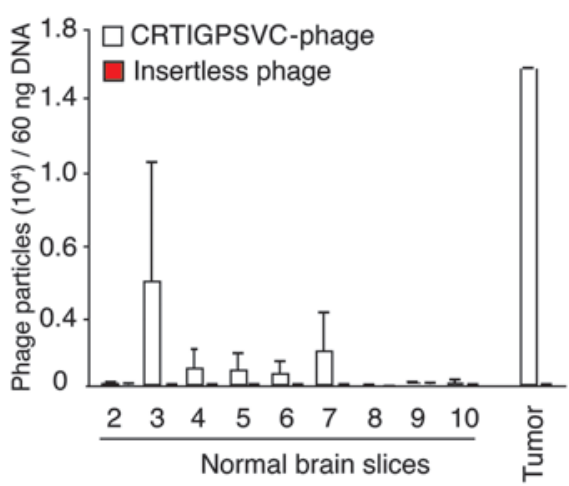

Figure 3

In vivo targeting of Tf/TfR. (A) Schematic representation of the brain slicing technique and quantitative real-time PCR used to demonstrate that CRTIGPSVC-phage crosses the BBB. In step 1, phage was injected i.v. into tail veins of mice. In step 2, brain and control organs were collected. Coronal brain slices, 1-mm thick, were obtained with the aid of a brain-slicer mold. H\&E-stained sections illustrate prominent tissue components: the lateral ventricle (LV; slice 2), hippocampus (Hip; slices 4 and 6), and cerebellum (primary fissure [prf]; slice 9). Insets show larger views; the boxes represent the area shown in high magnification in the larger images. In step 3, total DNA of each brain slice was purified and used as template for phage DNA amplification and quantification by quantitative real-time PCR. Original magnification, $\times 10 ; \times 4$ (insets). (B-D) Quantification of phage homing to tumor and normal brain after (B) 10 minutes, (C) 30 minutes, and (D) 1 hour of systemic circulation.

of phage particles was also detected in the tumor parenchyma, data demonstrating passage of CRTIGPSVC-phage from gliomaassociated blood vessels to tumor cells (Figure 4, B-E). Negative controls included non-targeted phage (Figure 4F) and unrelated regions of the brain, such as cerebral cortex (Figure 4G) and caudate/putamen (Figure 4H). Immunofluorescence staining of frozen tissue sections confirmed a widespread and homogeneous distribution of targeted phage to the tumor vasculature but not to the blood vessels of normal tissue (Figure 4, I-K). No signal was exhibited by insertless phage (Figure 4, L-N).
By confocal microscopy, we confirmed that the mechanism by which the CRTIGPSVC peptide targets and translocates into the tumor vasculature is indeed mediated by the Tf/TfR protein complex. Thick sections of frozen brain from tumor-bearing animals systemically receiving CRTIGPSVC-phage i.v. were immunostained for CD31 (Figure 4O), TfR (Figure 4P), and phage (Figure 4Q); TfR and phage particles were found to coincide in blood vessels (Figure 4R). There was also colocalization of CRTIGPSVC-phage and TfR in tumor parenchymal cells (Figure 4, S and T, arrows), consistent with our interpretation. 

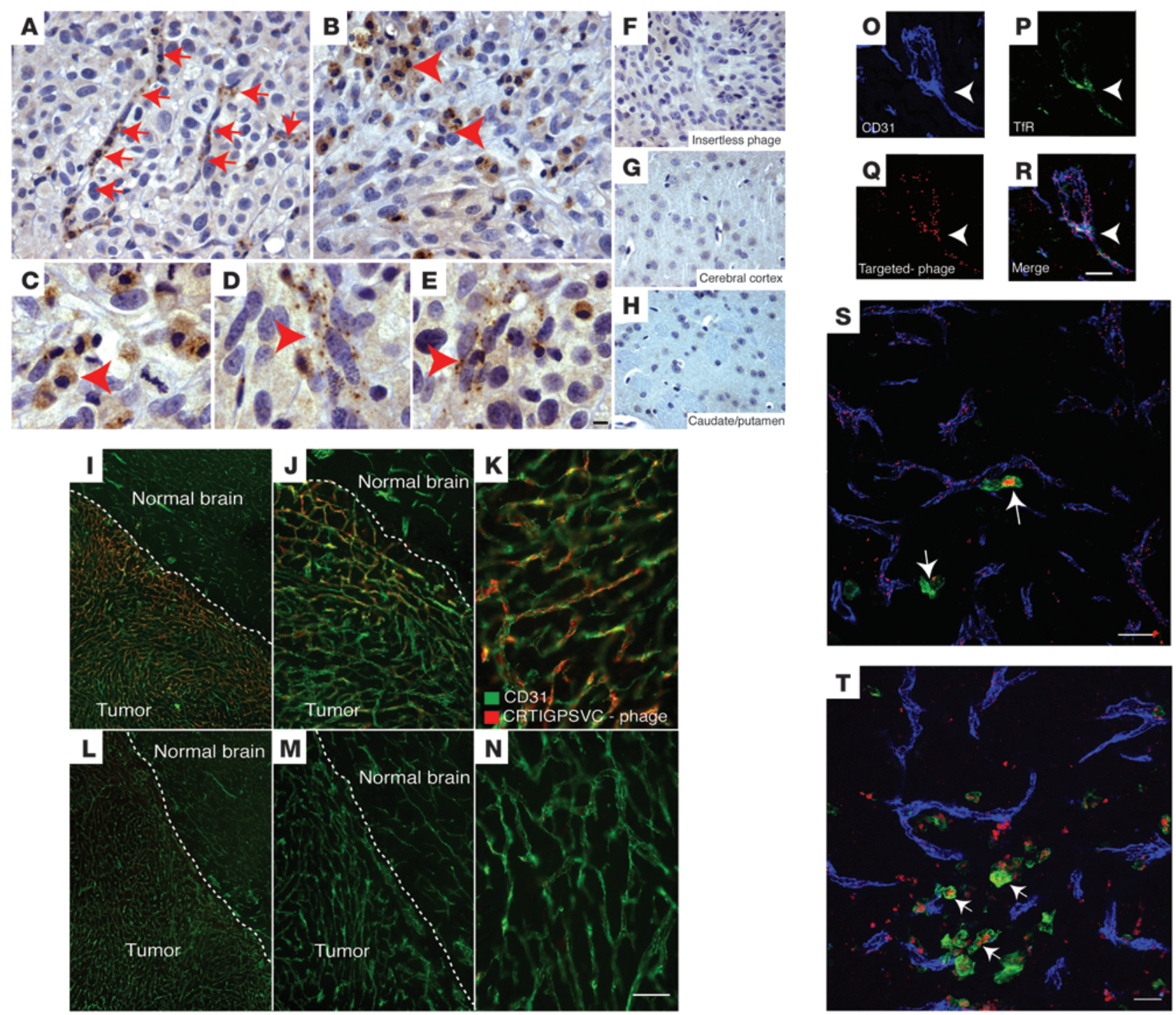

\section{Figure 4}

CRTIGPSVC-phage targets human glioblastoma in vivo. (A-H) Immunohistochemistry of CRTIGPSVC-phage after systemic circulation into human-derived glioblastoma xenograft-bearing mice. Staining of phage was observed in (A) tumor blood vessels and (B-E) glioma cells. Arrows indicate positive phage staining in blood vessels. Arrowheads indicate positive phage staining in glioma cells. (F) Insertless phage was used as a negative control. Other areas of the brain such as $(\mathbf{G})$ cerebral cortex and $(\mathbf{H})$ caudate/putamen showed only background staining. (I-N) Immunofluorescence of phage (red) and CD31 (green) revealed accumulation of phage particles in (I-K) tumor blood vessels but not in normal tissue. Dashed lines indicate the tumor circumference. (L-N) Insertless phage, used as a negative control, revealed little or no staining.

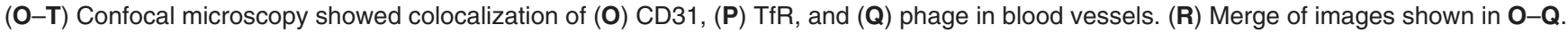
Arrowheads point to protein colocalization. ( $\mathbf{S}$ and $\mathbf{T}$ ) Phage particles were also found in TfR-expressing cells in the tumor stroma (arrows). Scale bars: $100 \mu \mathrm{m}(\mathbf{A}-\mathbf{N}) ; 20 \mu \mathrm{m}(\mathbf{O}-\mathbf{T})$.

Targeted transgene delivery and molecular imaging of brain tumors. We showed that CRTIGPSVC-phage preferentially targets TfR-expressing cells and demonstrated that transport of phage into normal brain and brain tumor vasculature is mediated by the Tf/TfR molecular complex. Next, to begin to examine the translational potential of this discovery, we generated a CRTIGPSVC-targeted AAVP vector (20-23) to merge gene delivery and molecular imaging in an orthotopic preclinical model of human glioma. A CRTIGPSVC-targeted AAVP construct carrying the herpes simplex virus thymidine kinase gene (HSV-TK) was engineered; the HSV-TK transgene can serve as both a genetic suicide strategy when combined with drugs, such as ganciclovir (GCV), or as a reporter for molecular-genetic imaging of tracers, such as $\left[{ }^{18} \mathrm{~F}\right]$-FEAU. We delivered CRTIGPSVC-targeted AAVP-HSV-TK or control insertless AAVP-HSV-TK to cohorts of brain tumor-bearing mice (Figure 5). Animals treated with vehicle were also used as controls ( $n=10$, each group). Both targeted and untargeted groups were treated with GCV, and response to treatment was assessed by measurement of tumor areas (Figure 5A) and by histopathological examination (Figure 5, E and F). After GCVbased therapy, the size of tumors in mice that received CRTIGPSVC AAVP-HSV-TK was significantly smaller (Student's $t$ test, $P<0.001$ ) than that in mice receiving non-targeted AAVP-HSV-TK or vehicle 


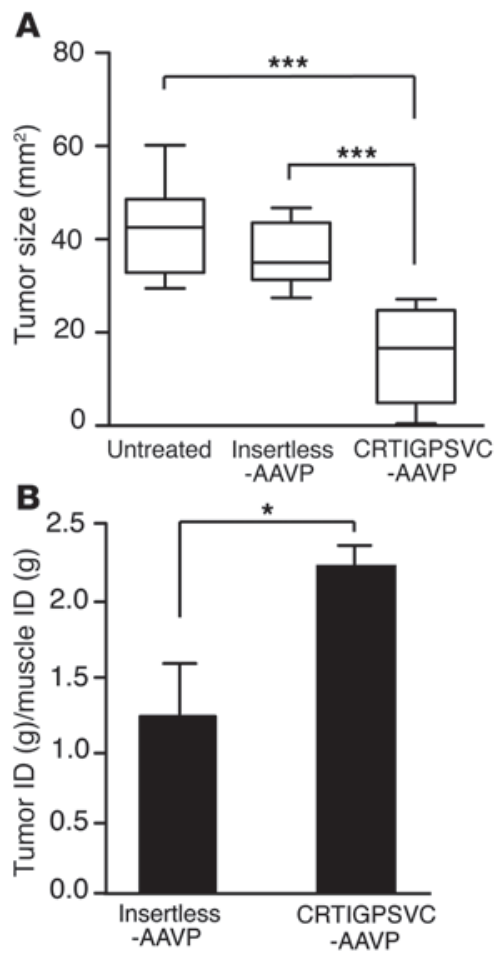

$\mathbf{E}$

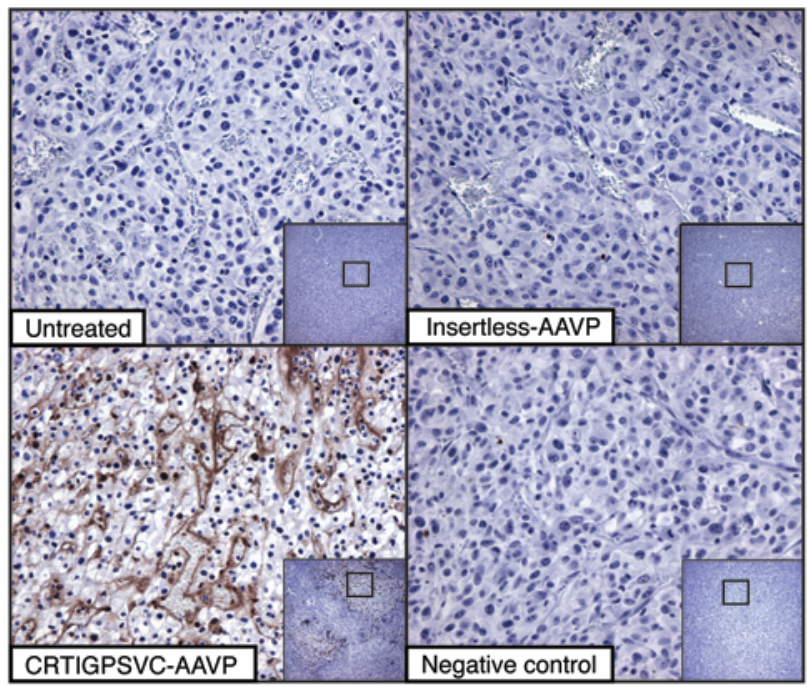

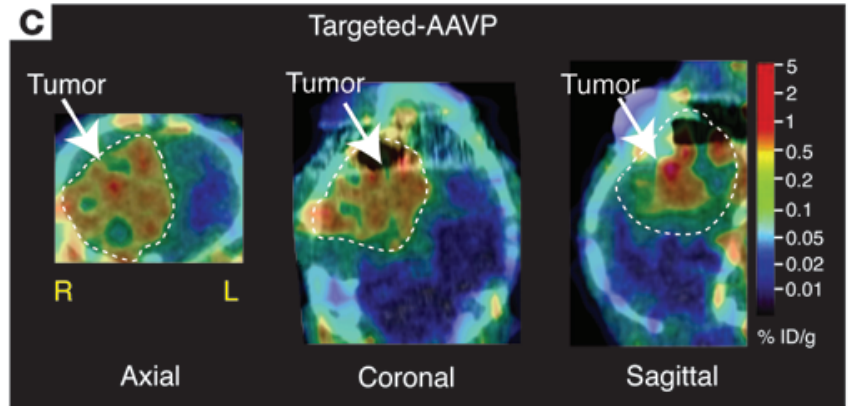

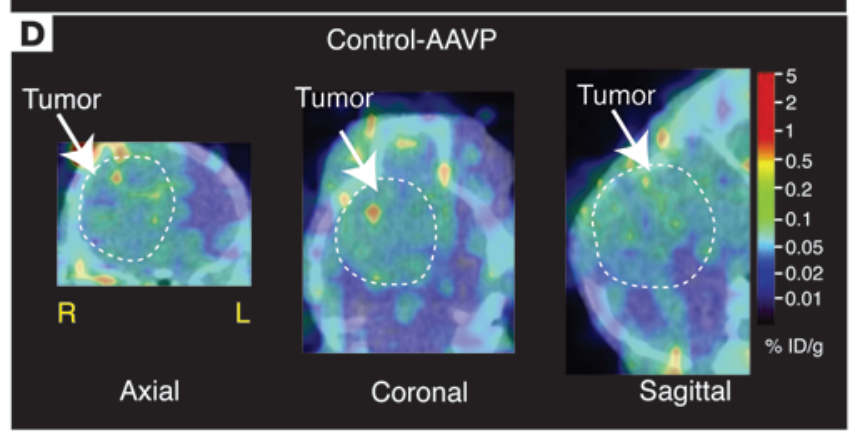

$\mathbf{F}$

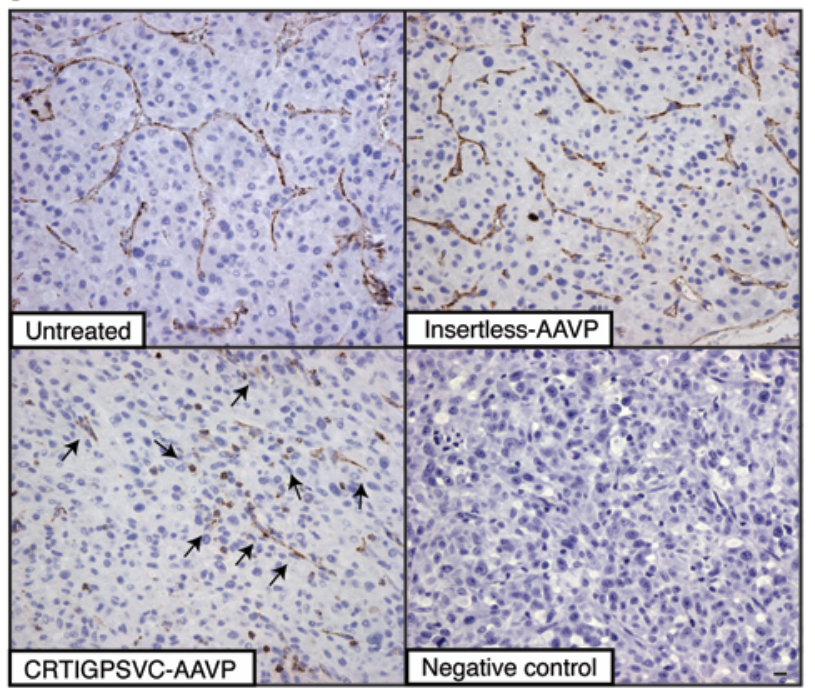

\section{Figure 5}

Targeted therapy and molecular-genetic imaging of intracranial brain tumors in an orthotopic model of human-derived glioblastoma xenograft. (A) Tumor growth suppression by CRTIGPSVC AAVP-HSV-TK transduction plus treatment with GCV. Cohorts of immunodeficient nude mice $(n=10)$ with established intracranial tumors received a single systemic administration of targeted CRTIGPSVC AAVP-HSV-TK $\left(5 \times 10^{10}\right.$ TU) or control AAVP-HSV-TK. Mice received GCV from day 7 after systemic i.v. AAVP administration until the end of the experiment (daily GCV dose i.p. for 5 days). Untreated group received vehicle alone. The boxes represent the upper (25\% of data greater than this value) and lower (25\% of data less than this value) quartiles. Medians are indicated by horizontal lines; whiskers represent the maximum and minimum values of the data set. ${ }^{*} P<0.05 ;{ }^{* * *} P<0.001$. (B) Temporal dynamics of $H S V$-TK gene expression as assessed by micro-PET imaging with $\left[{ }^{18} \mathrm{~F}\right]-\mathrm{FEAU}$. ${ }^{*} P<0.05$. (C and D) Combined PET/CT images of brain tumor-bearing animals, systemically administered with (C) targeted CRTIGPSVC AAVP-HSV-TK or (D) control insertless AAVP-HSV-TK. Axial, coronal, and sagittal planes are shown. [18$\left.{ }^{18} \mathrm{~F}\right]$-FEAU uptake was observed only in animals receiving targeted AAVP. Arrows point to the tumor area. Dashed lines indicate the tumor circumference. R, right; L, left. (E) Detection of apoptotic cells by TUNEL was performed on tumor tissue sections of animals treated with control insertless AAVP or with targeted AAVP. Untreated animals received only vehicle. Nonimmune IgG was used as negative control. Insets of serial tumor sections show the area containing the larger views; the boxes represent the area shown in high magnification in the larger images. (F) CD31 staining was performed on paraffin-embedded brain tumor tissue sections of animals treated with targeted AAVP or control AAVP. Untreated animals received only vehicle. Arrows point to disrupted blood vessels. Tumor tissue stained with IgG control isotype was used as negative control. Scale bar: $200 \mu \mathrm{m}$. 
A
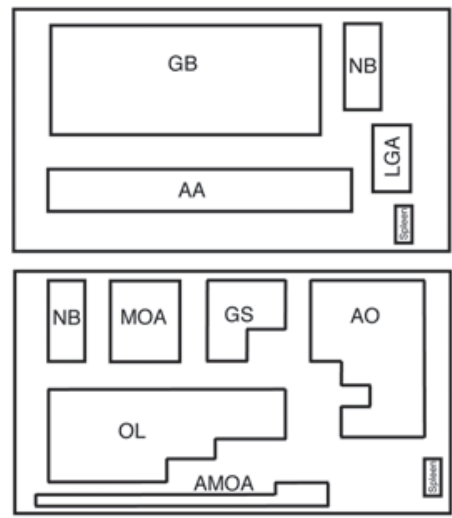
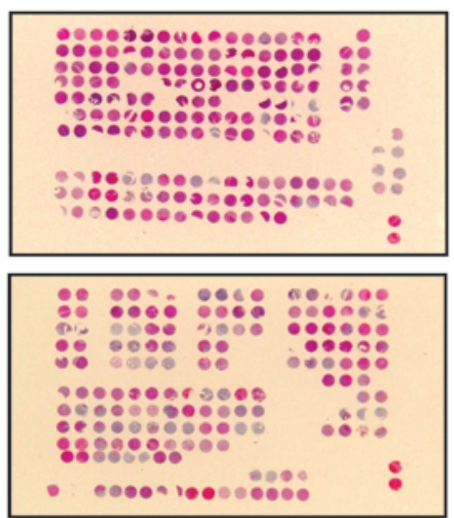

D

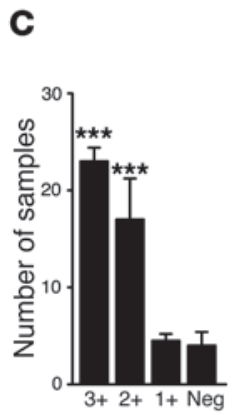

E

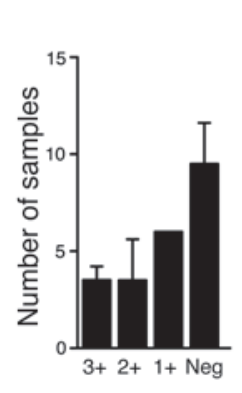

Glioblastoma multiforme $(n=49)$

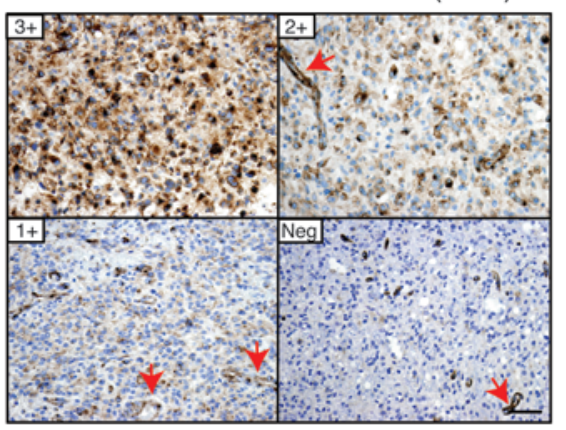

Anaplastic astrocytoma $(n=23)$

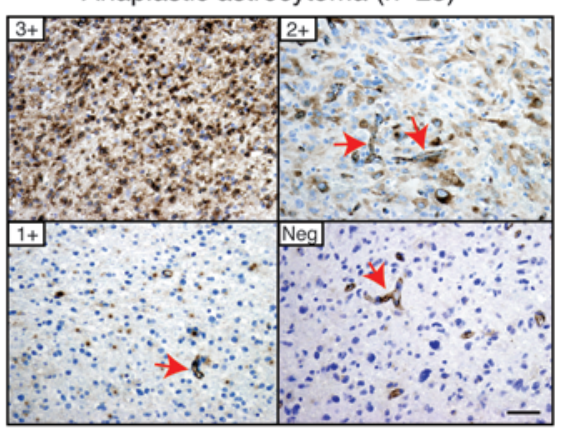

B

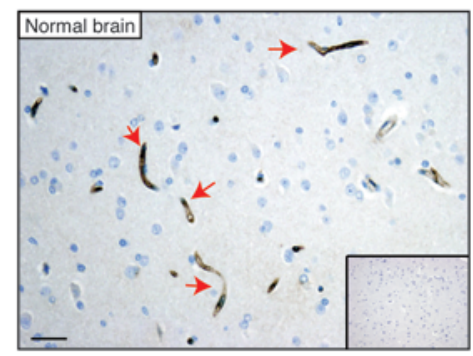

Gliosarcoma $(n=6)$

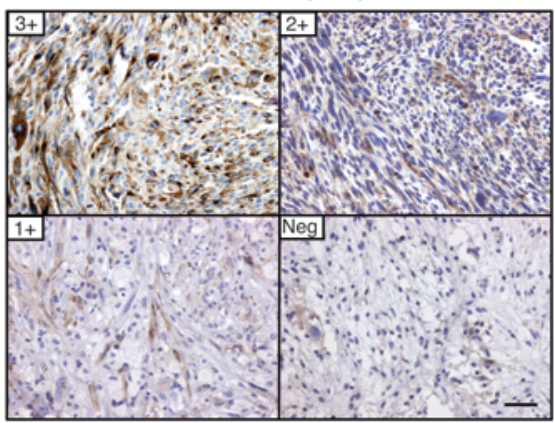

Oligodendroglioma $(n=22)$

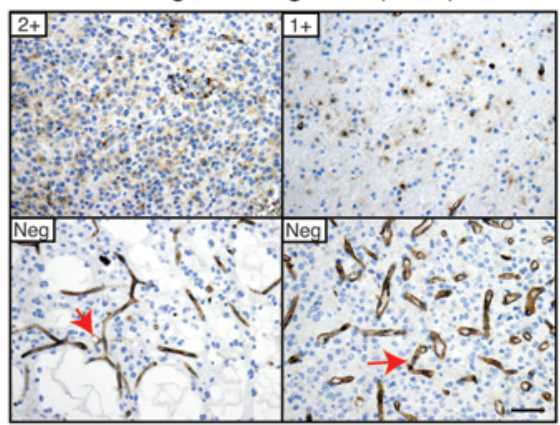

Figure 6

Evaluation of TfR expression in comprehensive human glioma TMA. (A and B) Glioma TMA maps and H\&E-stained tissue sections. AA, anaplastic astrocytoma; $\mathrm{AO}$, anaplastic oligodendroglioma; $\mathrm{AMOA}$, anaplastic mixed oligoastrocytoma; GM, glioblastoma multiforme; GS, gliosarcoma; LGA, low-grade diffuse astrocytoma; MOA, mixed oligoastrocytoma; NB, normal brain; and OL, oligodendroglioma. (B) The inset represents negative control. Arrows point to TfR-positive blood vessels. (C-F) TfR expression in (C) glioblastoma multiforme, (D) gliosarcoma, (E) anaplastic astrocytoma, and (F) oligodendroglioma. Representative photomicrographs are shown. Pathology scores for tumor cells are as follows: $3+$, strong; $2+$, moderate, $1+$, weak. Arrows point to blood vessels. Scale bar: $100 \mu \mathrm{m}$. ${ }^{\star \star \star} P<0.001$ (tumor vs. normal brain).

alone (Figure 5A). Next, we tested the efficacy of clinically applicable molecular-genetic PET imaging with [18 F]-FEAU combined with CT scan imaging for noninvasive monitoring of temporal dynamics and spatial heterogeneity of $H S V-T K$ reporter gene expression in living orthotopic glioma-bearing mice. PET imaging with $\left[{ }^{18} \mathrm{~F}\right]$-FEAU was performed 7 and 10 days after vector administration to assess localization and magnitude of HSV-TK expression. No specific transgene expression was detected 7 days after AAVP administration i.v. (data not shown). In contrast, we observed significant (Student's $t$ test, $P<0.05$ ) levels of HSV-TK expression (expressed as relative tumor injected dose $[\mathrm{g}] /$ muscle injected dose [g]; i.e., tumor/muscle ID/g) after 10 days in the tumors of animals administered with CRTIGPSVC AAVP-HSV-TK compared with those in the control group (Figure 5B). Combined micro-PET/CT images in axial, coronal, and sagittal planes in tumor-bearing mice revealed heterogeneous targeting of intracranial human glioblastoma xenografts with little or no signal in the normal brain tissue (Figure 5C), whereas control insertless AAVP yielded negative results in both normal brain and tumor (Figure 5D). Finally, TUNEL staining clearly demonstrated apoptosis of endothelial and glioma cells heterogeneously distributed within the tumor, but no apoptosis was observed in tumors from mice 
that received either non-targeted AAVP-HSV-TK or vehicle alone (Figure 5E). No apoptosis above background was detected in normal brain tissue (Supplemental Figure 6). Immunohistochemical studies for CD31 expression demonstrated that systemic administration of CRTIGPSVC AAVP-HSV-TK followed by 5 daily i.p. doses of GCV caused targeted destruction of capillary-like blood vessels, characteristic of experimental models of glioblastoma multiforme. This effect was not observed in control animals (Figure 5F). Together, these results demonstrate that the level of $H S V-T K$ transgene expression in human glioblastoma xenografts is sufficient for effective GCV activation.

In order to begin to assess the feasibility of this preclinical strategy for potential clinical applications, we used high-throughput glioma TMA samples to analyze the expression of TfR in a large $(n=165)$ and comprehensive panel of primary human gliomas, with well-annotated clinical and pathological features (Figure 6A). Strong $(3+)$ and moderate $(2+)$ TfR staining was observed in tumor cells in $85 \%$ of analyzed glioblastoma samples (Figure 6C). Positive staining was also detected in the majority of gliosarcomas and anaplastic astrocytomas (Figure 6, D and E). In contrast, tumor cells were negative within the limits of our immunohistochemical procedures in low-grade oligodendrogliomas (Figure 6F), anaplastic mixed oligoastrocytomas, mixed oligoastrocytomas, and anaplastic oligodendrogliomas (data not shown). Notably, the small blood vessels were positive for TfR not only in tumor samples but also in normal brain (Figure 6B, arrows). This patient-derived data set indicates that TfR might indeed be a suitable candidate target for translation into clinical applications.

\section{Discussion}

Development of effective treatments against tumors of the CNS requires the identification of targets to enable access to this sanctuary site. For many decades, the potential advantage of therapeutically exploiting receptor-mediated transporters of the BBB has been pursued, and the TfR has long been considered among the most promising targets. However, while a large body of previous work with monoclonal antibodies against the TfR demonstrated preclinical efficacy in animal models, translation into clinical applications met with far less effective results.

Here, we introduce a supervised combinatorial approach to target receptor-mediated transporters of the BBB in vivo. This peptide-based strategy provides an alternative platform capable of breaching the BBB. In a model of human glioma, a combination of TfR overexpression plus extended vascular permeability and ligand retention results in effective targeting.

Differences in the delivery of CRTIGPSVC-targeted AAVP to normal mouse brain versus the grafted human brain tumors are likely influenced not only by the higher TfR distribution and accessibility in the tumor vascular and parenchymal cells but also perhaps by other parameters, such as circulation time, endothelial permeability, hydrostatic pressure, and receptor turnover rate. This combination of multiple factors, along with the biodistribution studies performed in vivo, support the observation that targeted CRTIGPSVC-phage homes specifically to tumor sites relative to normal organs. Analysis of human samples suggests a translational approach for the detection and treatment of gliomas. One should also note that human and mouse Tf and TfR are more than $70 \%$ conserved (48); thus, species of origin (i.e., mouse vs. human) is unlikely to influence the targeting properties of the CRTIGPSVC peptide in this experimental system.
The key evidence that CRTIGPSVC-phage crosses from brain blood vessels into brain parenchyma is shown in Figure 1E. We demonstrate that control phage is present only in the blood vessel fraction but not in the brain parenchyma fraction, an indication that the parenchyma fraction is not heavily contaminated by vessels. The corresponding targeted phage distribution is dramatically different, showing extensive passage of phage carrying the targeting peptide across blood vessels and into brain parenchyma. However, this fundamental point requires future confirmation by independent methods. Further, we have not yet determined the full kinetics of binding competition between the apo-Tf/CRTIGPSVC peptide complex and holo-Tf in the bloodstream and whether there is significant passage of AAVP or phage vectors from normal brain endothelial cells to parenchymal cells in the first minute after administration. Future $\mathrm{x}$-ray crystallography studies might shed additional light into the mechanism of binding of this protein complex.

Targeted delivery of agents from the circulation into intact CNS or into brain tumors thus appears to have unequivocal translational relevance. The design and validation of a clinic-ready, AAVPbased targeting trans-BBB approach may open the prospect of visualizing brain tumor presence and exact location for diagnosis and serially quantifying spatial changes in response to therapy in real time. Moreover, one might predict that either genetic disorders globally affecting the CNS (i.e., lysosomal storage and myelin diseases) or degenerative diseases targeted on specific neuronal populations may also benefit from the attributes of peptide CRTIGPSVC, which, in principle, allows selective delivery of the agents, without simultaneous opening of the BBB to potentially harmful blood-derived molecules. Indeed, the very property of selective opening of the BBB precludes assaying the efficiency of targeted AAVP delivery by the standard methods of measuring transfer of tracers from the blood into CNS parenchyma. Finally, given our human TMA results, the translation of the functional features of peptide-directed particles from animal models into clinical applications may hold promise for the targeted molecular imaging and therapy of brain tumors in cancer patients.

\section{Methods}

Animals. We used 8-week-old female nude $(n u / n u)$ mice and BALB/c mice housed in the animal facilities of The University of Texas M.D. Anderson Cancer Center or the Ludwig Institute for Cancer Research. All animal procedures were reviewed and approved by the Institutional Animal Care and Use Committees of these institutions.

Reagents. The following antibodies were used: goat polyclonal anti-TfR IgG (R\&D Systems), mouse monoclonal anti-TfR IgG (Fisher Scientific), rabbit polyclonal anti-bacteriophage (Sigma-Aldrich), rat monoclonal anti-CD31 (BD Pharmingen), and rabbit polyclonal anti-CD31 (AbCam). Secondary HRP-conjugated anti-rabbit IgG, Cy-3-conjugated anti-rabbit IgG, Cy-5conjugated anti-hamster IgG, and FITC-conjugated anti-rat IgG were purchased from Jackson ImmunoResearch Laboratories Inc. Human TfR (>95\% purity) and FragEL were purchased from Calbiochem, and human apo $(>90 \%$ purity) and holo-Tf (>90\% purity) were purchased from R\&D Systems. All synthetic peptides were synthesized and cyclized according to our specifications by commercial vendors (AnaSpec or PolyPeptide Laboratories).

Cell culture. U87-MG human glioblastoma cells (ATCC), rat gliosarcoma cells (9L) (31), and rat gliosarcoma cells expressing human TfR (9L 3.9) (31) were maintained in DMEM supplemented with $10 \%$ FBS and antibiotics.

Phage display selection and phage homing in vivo. In vivo phage screening was performed as described previously (26). Animals received $10^{10} \mathrm{TU}$ phage library i.v., and brains were collected after 24 hours of circulation. Bound 
phage were recovered by bacterial infection and were processed as described previously (26). For homing of selected phage in vivo, animals $(n=5$, each group) were deeply anesthetized and received $10^{10} \mathrm{TU}$ targeted phage or insertless control phage i.v. After 10 minutes, 30 minutes, 6 hours, or 24 hours, animals were perfused through the heart with $10 \mathrm{ml}$ DMEM.

Isolation of brain blood vessels. Fractionation of brain blood vessels was performed as described previously $(29,30)$. Mice aged 3-5 weeks were deeply anesthetized and perfused through the heart with $10 \mathrm{ml}$ PBS. Brain tissue homogenate was diluted to a final volume of $5 \mathrm{ml}$, transferred to the top of a $30 \%(\mathrm{w} / \mathrm{v})$ dextran solution, and centrifuged for 25 minutes at $3,000 \mathrm{~g}$ $\left(4^{\circ} \mathrm{C}\right)$. The myelin layer and pellet containing blood vessels were carefully collected. The final fractions were resuspended in an adequate volume and used for DNA or RNA extraction.

$D N A$ and RNA isolation from tissue and quantitative real-time PCR. Total DNA was isolated according to manufacturer's instructions (DNeasy Blood and Tissue Kit, Qiagen). The amount of phage present in each tissue sample was determined by quantitative PCR (7500 Fast Real-Time PCR System instrument, Applied Biosystems), as described previously (49). Each point of the curve, as well as each tissue sample of DNA, was determined in triplicate. For gene expression studies, 2 sets of total RNA were independently isolated from microvessel and parenchyma fractions with the RNeasy Mini Kit (Qiagen). DNA synthesis was performed with the SuperScript III FirstStrand Synthesis System (Invitrogen). The following primer pairs were used: slc2a1 (glut-1), 5' TTCTCTGTCGGCCTCTTTGT 3' and 5'AGGCCAACAGGTTCATCATC 3'; VE-cadherin, 5' TCATCAAACCCACGAAGTCC $3^{\prime}$ and 5' TGTTTTTGCCTGAAGTGCTG 3'; Occludin: 5' CCTACTCCTCCAATGGCAAA $3^{\prime}$ and 5' GGCACCAGAGGTGTTGACTT $3^{\prime}$.

High-density TMAs. The construction of the human glioma TMA was previously described (50). The TMA included primary human gliomas, with all histologic subtypes and grades of diffuse gliomas as codified in the WHO classification (51), including GBM, AA, AO, O, AMOA, MOA, and GS. Lowgrade astrocytomas were not analyzed here, because of their small sample size $(n=4)$. Pathology scoring was performed by 2 independent observers (F.I. Staquicini and G.N. Fuller).

Immunohistochemistry. Immunohistochemistry on sections of fixed, paraffin-embedded mouse tissue was performed with a labeled streptavidin biotin (LSAB) plus peroxidase kit (DAKO). Slides were blocked for nonspecific protein binding, and a polyclonal rabbit anti-bacteriophage primary antibody was added (1:500 dilution), followed by 1-hour incubation with a peroxidaseconjugated anti-rabbit secondary antibody. Slides were developed with a specific substrate (chromogen DAB). Immunohistochemistry on glioma TMAs and CD31 staining were performed on an automated immunohistochemical autostainer (Lab Vision Corp.). Detection of apoptosis in paraffin-embedded specimens was performed with the FragEL DNA Fragmentation Detection Kit (Calbiochem). Hematoxylin was used for counterstaining.

Immunofluorescence. Immunofluorescence was performed on 1\% PFA-fixed cryostat sections (60- to $80-\mu \mathrm{m}$ thickness). Tissues were washed 3 times with PBS and once with PBS containing $0.3 \%$ Triton X-100, followed by blocking for 1 hour in $5 \%$ appropriate normal serum diluted in PBS containing $0.3 \%$ Triton X-100. Tissue sections were incubated with specific antibodies diluted in PBS containing 1\% appropriate normal serum and $0.3 \%$ Triton $\mathrm{X}-100$ for 1 hour at room temperature. Sections were stained for 1 hour with Cy3-conjugated and FITC-conjugated secondary antibodies. Confocal images were acquired on a laser scanning confocal microscope (Zeiss LSM510) equipped with krypton-argon and helium-neon lasers. Image analysis was performed with the Zeiss LSM 3.2 software package.

In vitro phage binding assays. TfR, apo-Tf, holo-Tf, and BSA were immobilized on microtiter wells of 96 -well plates overnight $(\mathrm{ON})$ at $4{ }^{\circ} \mathrm{C}$. Wells were blocked with PBS containing 3\% BSA for 1 hour at room temperature and incubated with $10^{9} \mathrm{TU}$ phage. Bound phage were recovered by infec- tion of host bacteria with $200 \mu \mathrm{l}$ E. coli K91 Kan in log phase. To test phage binding inhibition by free iron, we used an initial solution of $10 \mathrm{mM}$ iron (III) phosphate $\left(\mathrm{FePO}_{4}\right)$ (Sigma-Aldrich) in double-distilled water. Final concentrations of iron ranged from $0.01 \mathrm{mM}$ to $0.5 \mathrm{mM}$.

Phage binding assays on cells. For cell-phage binding assays, $10^{6}$ cells were incubated with $10^{9} \mathrm{TU}$ phage for 2 hours on ice in the presence of either apo- or holo-Tf $(200 \mu \mathrm{g} / \mathrm{ml})$ in $200 \mu \mathrm{l}$ DMEM. Cells and phage were centrifuged through the organic phase, and the cell-bound phage were recovered by bacterial infection of host $E$. coli as described previously (52).

Steady-state fluorescence spectroscopy. Steady-state tryptophan fluorescence spectra were obtained on a Spectrofluorimeter LS 50B (Perkin-Elmer). Increasing molar ratios of $\mathrm{Fe}^{+3}$, the targeted peptide CRTIGPSVC, or control peptides (control peptide 1, CGLPYSSVC; control peptide 2, CSGIGSGGC; control peptide 3, CRFESSGGC; and control peptide 4, CPQRGVTPC) were incubated $\mathrm{ON}$ at room temperature with $2 \mu \mathrm{M}$ apo-Tf in buffer containing $100 \mathrm{mM}$ HEPES, $10 \mathrm{mM} \mathrm{NH}_{4} \mathrm{CO}_{3}, \mathrm{pH}$ 7.4. Samples were excited at $295 \mathrm{~nm}$, and emission scans were collected from 305 to $400 \mathrm{~nm}$, with an excitation slit of $2.5 \mathrm{~nm}$ and an emission slit of $6 \mathrm{~nm}$. All spectra were corrected for background fluorescence by subtraction of the appropriate blanks.

$C D$. CD spectra were recorded on a Jasco $J 720$ spectropolarimeter from 230 to $320 \mathrm{~nm}$, with a bandwidth of $1 \mathrm{~nm}$, and integrated for 1 second at $0.2-\mathrm{nm}$ intervals. Samples were measured at room temperature using cuvettes with a 2-mm path length. Absorbance values of $\mathrm{Fe}^{+3}$ and peptide alone were subtracted from the results. Increasing concentrations of $\mathrm{Fe}^{+3}$, the targeted peptide CRTIGPSVC, or control peptides (control peptide 1, CGLPYSSVC; control peptide 2, CSGIGSGGC; control peptide 3, CRFESSGGC; and control peptide 4, CPQRGVTPC) were titrated in $100 \mu \mathrm{M}$ apo-Tf in buffer containing $100 \mathrm{mM}$ HEPES, $10 \mathrm{mM} \mathrm{NH}_{4} \mathrm{CO}_{3}$, $\mathrm{pH}$ 7.4. The data were expressed as mean residue ellipticity $(\theta)$ in $\mathrm{deg} \mathrm{cm}^{2} / \mathrm{dmol}$, which was calculated from $(\theta)=(d \times s \times M) /(c \times l)$, where $d$ denotes observed ellipticity (the displacement in $\mathrm{cm}$ from the baseline), $s$ denotes sensitivity in $\mathrm{mdeg} / \mathrm{cm}, M$ denotes the mean residue weight, $c$ denotes protein concentration in $\mathrm{mg} / \mathrm{ml}$, and $l$ denotes cell path length. The concentrations of protein were kept at $8 \mathrm{mg} / \mathrm{ml}$.

Orthotopic human glioblastoma xenografts. We used a guide-screw system to implant human glioma cells into the mouse brain (41). Animals were kept warm until their recovery from anesthesia and were allowed to move around freely thereafter. In vivo homing experiments with targeted and control phage were performed approximately $12-15$ days after tumor implantation.

Subcutaneous human glioblastoma xenografts. A solution containing $3 \times 10^{6}$ U87-MG cells was subcutaneously injected into the right flank of nude mice $(n=5$, each group). Tumor homing experiments were performed when palpable subcutaneous tumor xenografts reached 6-8 $\mathrm{mm}$ in diameter.

Targeted therapy and molecular-genetic imaging. Orthotopic brain tumor-bearing animals received a single dose ( $10^{11} \mathrm{TU}$ i.v. per mouse) of CRTIGPSVC AAVP-HSV-TK or control, approximately 7 days after tumor implantation. Treatment with GCV $(80 \mathrm{mg} / \mathrm{kg} / \mathrm{d}$ i.p.) was initiated 7 days after AAVP administration i.v. To image HSV-TK expression, PET and CT scans were performed 2 hours after i.v. administration of the radiolabeled nucleoside analog $\left[{ }^{18} \mathrm{~F}\right]$-FEAU. A microPET R4 (Concorde Microsystems), equipped with a computer-controlled positioning bed in a $10.8-\mathrm{cm}$ transaxial and 8 -cm axial field of view with no septa and operating in 3D list mode, was used. PET/CT imaging was performed with an Inveon micro-PET/CT scanner (Siemens Preclinical Solution). Glioma-bearing mice were anesthetized (with isoflurane $2 \%$ in $98 \%$ oxygen), and their temperature was kept at $38^{\circ} \mathrm{C}$ with a heat lamp. The microCT imaging parameters were as follows: $\mathrm{x}$-ray voltage of $80 \mathrm{kVp}$, anode current of $500 \mu \mathrm{A}$, and exposure time of 300-350 milliseconds for each of the 360 rotational steps. Images were reconstructed by a $2 \mathrm{D}$ ordered subsets expectation maximization algorithm. PET and CT image fusion and image analysis were performed with vendor software ASIPro 5.2.4.0 (Siemens Preclinical Solution). 
Radiolabeled substrate synthesis. Radiolabeled $\left[{ }^{18} \mathrm{~F}\right]$-FEAU was synthesized to radiochemical purity of more than $99 \%$ by using 5 -ethyluracil2,5-bis-trimethylsilyl ether as the pyrimidine base for condensation with 1-bromo-2-deoxy-2-[18F]fluoro-3,5-di-O-benzoyl- $\alpha$-D-arabinofuranose. For quantification of $\left[{ }^{18} \mathrm{~F}\right]$-FEAU radioactivity, regions of interest were drawn on images, and the measured values were converted from $\mathrm{nCi} / \mathrm{mm}^{3}$ into percentage of injected dose per gram of tissue (\% ID/g) $(22,53)$.

Statistics. All numerical data are expressed as mean \pm SEM. We analyzed data sets for significance with Student's $t$ test and 2-way ANOVA. We considered $P$ values of less than 0.05 as statistically significant.

\section{Acknowledgments}

We thank E. Helene Sage for critical reading of the manuscript; Richard G. Brennan, Emmanuel Dias-Neto, and Rebecca RichardsKortum for helpful discussions; and David Bier for graphic design. This work was supported by grants from the National Institutes of Health (to R. Pasqualini and W. Arap), National Cancer Institute (to R. Pasqualini, W. Arap, and W.K. Cavenee), and Department of Defense (to R. Pasqualini and W. Arap) and by awards from the Gillson-Longenbaugh Foundation, AngelWorks, and the Marcus Foundation (to R. Pasqualini and W. Arap). O. Bogler was supported by a grant from the Center for Targeted Therapy of The University of Texas M.D. Anderson Cancer Center. W.K. Cavenee was supported by a grant from the National Foundation for Cancer Research. C.A. Moya received pre-doctoral fellowships from the Harry S. and Isabel C. Cameron Foundation and the Schissler Foundation.

Received for publication August 17, 2010, and accepted in revised form October 27, 2010.

Address correspondence to: Renata Pasqualini or Wadih Arap, David H. Koch Center, The University of Texas M.D. Anderson Cancer Center, 1515 Holcombe Boulevard, Houston, Texas, USA. Phone: 713.792.3872; Fax: 713.745.2999; E-mail: rpasqual@mdanderson. org (R. Pasqualini); warap@mdanderson.org(W. Arap).

James P. Basilion's present address is: NFCR Center for Molecular Imaging at Case, Case Western Reserve University, Cleveland, Ohio, USA.

Hiroyuki Nishimori's present address is: Center for Gastroenterology, Teine Keijinkai Hospital, Teine-ku, Sapporo, Japan.
1. Davies OL, Hurst EW. Studies on the blood-brain barrier; the basis of dosage for animals of various weights. BrJ Pharmacol Chemother. 1948;3(4):309-314.

2. Onesti SJ. The blood-brain barrier. McGill Med J. 1950;19(4):203-210.

3. Zlokovic BV. The blood-brain barrier in health and chronic neurodegenerative disorders. Neuron. 2008;57(2):178-201.

4. Pardridge WM. Re-engineering biopharmaceuticals for delivery to brain with molecular Trojan horses. Bioconjug Chem. 2008;19(7):1327-1338.

5. Pardridge WM. Drug and gene delivery to the brain: the vascular route. Neuron. 2002;36(4):555-558.

6. Pardridge WM. Blood-brain barrier delivery. Drug Discov Today. 2007;12(1-2):54-61.

7. Abbott NJ, Ronnback L, Hansson E. Astrocyteendothelial interactions at the blood-brain barrier. Nat Rev Neurosci. 2006;7(1):41-53.

8. Banks WA. Are the extracellular [correction of extracelluar] pathways a conduit for the delivery of therapeutics to the brain? Curr Pharm Des. 2004;10(12):1365-1370.

9. Dogrukol-Ak D, et al. Isolation of peptide transport system- 6 from brain endothelial cells: therapeutic effects with antisense inhibition in Alzheimer and stroke models. J Cereb Blood Flow Metab. 2009;29(2):411-422.

10. Neuwelt E, et al. Strategies to advance translational research into brain barriers. Lancet Neurol. 2008;7(1):84-96.

11. Pardridge WM. Drug targeting to the brain. Pharm Res. 2007;24(9):1733-1744.

12. Banks WA. Delivery of peptides to the brain: emphasis on therapeutic development. Biopolymers. 2008;90(5):589-594.

13. Broadwell RD, Balin BJ. Endocytic and exocytic pathways of the neuronal secretory process and trans-synaptic transfer of wheat germ agglutinin-horseradish peroxidase in vivo. J Comp Neurol. 1985;242(4):632-650

14. Friden PM, Walus LR, Musso GF, Taylor MA, Malfroy B, Starzyk RM. Anti-transferrin receptor antibody and antibody-drug conjugates cross the blood-brain barrier. Proc Natl Acad Sci U S A. 1991;88(11):4771-4775.

15. Spencer BJ, Verma IM. Targeted delivery of proteins across the blood-brain barrier. Proc Natl Acad SciUS A. 2007;104(18):7594-7599.

16. Pardridge WM, Buciak JL, Friden PM. Selective transport of an anti-transferrin receptor antibody through the blood-brain barrier in vivo. J Pharmacol Exp Ther. 1991;259(1):66-70.

17. Boado RJ, Zhang Y, Wang Y, Pardridge WM. Engineering and expression of a chimeric transferrin receptor monoclonal antibody for blood-brain barrier delivery in the mouse. Biotechnol Bioeng. 2009;102(4):1251-1258

18. Zhang Y, Boado RJ, Pardridge WM. Marked enhancement in gene expression by targeting the human insulin receptor. J Gene Med. 2003;5(2):157-163.

19. Wu D, Pardridge WM. Pharmacokinetics and blood-brain barrier transport of an anti-transferrin receptor monoclonal antibody (OX26) in rats after chronic treatment with the antibody. Drug Metab Dispos. 1998;26(9):937-939.

20. Hajitou A, et al. A hybrid vector for ligand-directed tumor targeting and molecular imaging. Cell. 2006;125(2):385-398.

21. Hajitou A, et al. Design and construction of targeted AAVP vectors for mammalian cell transduction. Nat Protoc. 2007;2(3):523-531.

22. Soghomonyan S, et al. Molecular PET imaging of HSV1-tk reporter gene expression using [18F]FEAU. Nat Protoc. 2007;2(2):416-423.

23. Hajitou A, et al. A preclinical model for predicting drug response in soft-tissue sarcoma with targeted AAVP molecular imaging. Proc Natl Acad Sci U S A. 2008;105(11):4471-4476.

24. Arap W, et al. Steps toward mapping the human vasculature by phage display. Nat Med. 2002; $8(2): 121-127$

25. Kolonin MG, et al. Ligand-directed surface profiling of human cancer cells with combinatorial peptide libraries. Cancer Res. 2006;66(1):34-40.

26. Pasqualini R, Ruoslahti E. Organ targeting in vivo using phage display peptide libraries. Nature. 1996;380(6572):364-366

27. MacGillivray RT, et al. Two high-resolution crystal structures of the recombinant $\mathrm{N}$-lobe of human transferrin reveal a structural change implicated in iron release. Biochemistry. 1998;37(22):7919-7928.

28. Cheng Y, Zak O, Aisen P, Harrison SC, Walz T. Structure of the human transferrin receptor-transferrin complex. Cell. 2004;116(4):565-576.

29. Triguero D, Buciak J, Pardridge WM. Capillary depletion method for quantification of blood-brain barrier transport of circulating peptides and plasma proteins. J Neurochem. 1990;54(6):1882-1888.

30. Boado RJ, Pardridge MM. A one-step procedure for isolation of poly $(\mathrm{A})+\mathrm{mRNA}$ from isolated brain capillaries and endothelial cells in culture. J Neurochem. 1991;57(6):2136-2139.

31. Moore A, Basilion JP, Chiocca EA, Weissleder R. Measuring transferrin receptor gene expression by NMR imaging. Biochim Biophys Acta. 1998;1402(3):239-249.

32. James NG, Berger CL, Byrne SL, Smith VC, MacGillivray RT, Mason AB. Intrinsic fluorescence reports a global conformational change in the N-lobe of human serum transferrin following iron release. Biochemistry. 2007;46(37):10603-10611.

33. Byrne SL, Mason AB. Human serum transferrin: a tale of two lobes. Urea gel and steady state fluorescence analysis of recombinant transferrins as a function of $\mathrm{pH}$, time, and the soluble portion of the transferrin receptor. J Biol Inorg Chem. 2009;14(5):771-781.

34. James NG, Byrne SL, Steere AN, Smith VC, MacGillivray RT, Mason AB. Inequivalent contribution of the five tryptophan residues in the C-lobe of human serum transferrin to the fluorescence increase when iron is released. Biochemistry. 2009;48(13):2858-2867.

35. Jeffrey PD, Bewley MC, MacGillivray RT, Mason $\mathrm{AB}$, Woodworth RC, Baker EN. Ligand-induced conformational change in transferrins: crystal structure of the open form of the $\mathrm{N}$-terminal half-molecule of human transferrin. Biochemistry. 1998;37(40):13978-13986

36. Shen ZM, Yang JT, Feng YM, Wu CS. Conformational stability of porcine serum transferrin. Protein Sci. 1992;1(11):1477-1484.

37. Thakurta PG, Choudhury D, Dasgupta R, Dattagupta JK. Tertiary structural changes associated with iron binding and release in hen serum transferrin: a crystallographic and spectroscopic study. Biochem Biophys Res Commun. 2004; 316(4):1124-1131.

38. Trowbridge IS, Domingo DL. Anti-transferrin receptor monoclonal antibody and toxin-antibody conjugates affect growth of human tumour cells. Nature. 1981;294(5837):171-173

39. Recht L, Torres CO, Smith TW, Raso V, Griffin TW. Transferrin receptor in normal and neoplastic brain tissue: implications for brain-tumor immunotherapy. J Neurosurg. 1990;72(6):941-945.

40. Prior R, Reifenberger G, Wechsler W. Transferrin receptor expression in tumours of the human nervous system: relation to tumour type, grading and tumour growth fraction. Virchows Arch A Pathol Anat 
Histopathol. 1990;416(6):491-496.

41. Lal S, Lacroix M, Tofilon P, Fuller GN, Sawaya R, Lang FF. An implantable guide-screw system for brain tumor studies in small animals. J Neurosurg. 2000;92(2):326-333.

42. Arap W, Pasqualini R, Ruoslahti E. Cancer treatment by targeted drug delivery to tumor vasculature in a mouse model. Science. 1998;279(5349):377-380.

43. Geier MR, Trigg ME, Merril CR. Fate of bacteriophage lambda in non-immune germ-free mice. Nature. 1973;246(5430):221-223.

44. Ozawa MG, et al. Beyond receptor expression levels: the relevance of target accessibility in liganddirected pharmacodelivery systems. Trends Cardiovasc Med. 2008;18(4):126-132.

45. Maeda $H$. The enhanced permeability and retention (EPR) effect in tumor vasculature: the key role of tumor-selective macromolecular drug targeting. Adv Enzyme Regul. 2001;41:189-207.

46. Iyer AK, Khaled G, Fang J, Maeda H. Exploiting the enhanced permeability and retention effect for tumor targeting. Drug Discov Today. 2006; 11(17-18):812-818.

47. Bartlett DW, Su H, Hildebrandt IJ, Weber WA, Davis ME. Impact of tumor-specific targeting on the biodistribution and efficacy of siRNA nanoparticles measured by multimodality in vivo imaging. Proc Natl Acad Sci U S A. 2007;104(39):15549-15554.

48. Baldwin GS. Comparison of transferrin sequences from different species. Comp Biochem Physiol. B. 1993;106(1):203-218.

49. Dias-Neto E, et al. Next-generation phage display: integrating and comparing available molecular tools to enable cost-effective high-throughput analysis. PLoS One. 2009;4(12):e8338.

50. Wang H, Zhang W, Fuller GN. Overexpression of IGFBP5, but not IGFBP3, correlates with the histologic grade of human diffuse glioma: a tissue microarray and immunohistochemical study. Technol Cancer Res Treat. 2006;5(3):195-199.

51. Louis DN, et al. The 2007 WHO classification of tumours of the central nervous system. Acta Neuropathol. 2007;114(2):97-109.

52. Giordano RJ, Cardó-Vila M, Lahdenranta J, Pasqualini R, Arap W. Biopanning and rapid analysis of selective interactive ligands. Nat Med. 2001;7(11):1249-1253.

53. Tjuvajev JG, et al. Imaging herpes virus thymidine kinase gene transfer and expression by positron emission tomography. Cancer Res. 1998; 58(19):4333-4341. 